\title{
SOLUTION OF UNSTEADY ROLLING MOTION OF SPHERES EQUATION IN INCLINED TUBES FILLED WITH INCOMPRESSIBLE NEWTONIAN FLUIDS BY DIFFERENTIAL TRANSFORMATION METHOD
}

\author{
Y. Rostamiyan ${ }^{1}$, S.D.Farahani ${ }^{2}$, M.R.Davoodabadi ${ }^{3}$ \\ ${ }^{1}$ Departments of Mechanical Engineering, Sari branch, \\ Islamic azad university, Sari, Iran \\ ${ }^{2}$ Departments of Mechanical Engineering, Tehran University, Tehran, Iran \\ ${ }^{3}$ Department of Mechanical Engineering, Semnan University, Semnan, Iran
}

\begin{abstract}
In this paper, the unsteady motion of a spherical particle rolling down an inclined tube in a Newtonian fluid for a range of Reynolds numbers was solved using a simulation method called the Differential Transformation Method (DTM). The concept of differential transformation is briefly introduced, and then we employed it to derive solution of nonlinear equation. The obtained results for displacement, velocity and acceleration of the motion from DTM are compared with those from numerical solution to verify the accuracy of the proposed method. The effects of particle diameter (size), continues phase viscosity and inclination angles was studied. As an important result it was found that the inclination angle does not affect the acceleration duration. The results reveal that the Differential Transformation Method can achieve suitable results in predicting the solution of such problems.
\end{abstract}

\section{KEYWORDS}

Spherical particle; Acceleration motion; Inclination angle; Non-linear equation, Differential Transformation Method (DTM).

\section{INTRODUCTION}

The description of the motion of immersed bodies in fluids is present in several manufacturing processes, e.g. sediment transport and deposition in pipe lines, alluvial channels, chemical engineering and powder process [1-6]. Several works could be found in technical literature which investigated the spherical particles in low and high concentration [7-9]. A particle falling or rolling down a plane in a fluid under the influence of gravity will accelerate until the gravitational force is balanced by the resistance forces that include buoyancy and drag. The constant velocity reached at that stage is called the "terminal velocity" or "settling velocity". Knowledge of the terminal velocity of solids falling in liquids is required in many industrial applications. Typical examples include hydraulic transport slurry systems for coal and ore transportation, thickeners,

${ }^{\star}$ Y.rostamiyaneyahoo.com

David C. Wyld et al. (Eds) : SAI, CDKP, ICAITA, NeCoM, SEAS, CMCA, ASUC, Signal - 2014

pp. 231-244, 2014. (C) CS \& IT-CSCP 2014

DOI : $10.5121 / \mathrm{csit} .2014 .41122$ 
mineral processing, solid-liquid mixing, fluidization equipment, drilling for oil and gas, geothermal drilling. The resistive drag force depends upon drag coefficient. Drag coefficient and terminal velocities of particles are most important design parameters in engineering applications. There have been several attempts to relate the drag coefficient to the Reynolds number. The most comprehensive equation set for predicting $C_{D}$ from $\mathrm{Re}$ for Newtonian fluids has been published by Clift et al. [10], Khan and Richardson [11], Chhabra [12] and Hartman and Yutes [13]. Comparing between most of these relationships for spheres, demonstrates quite low deviations [14].

The most of mentioned applications involve the description of the particle position, velocity and acceleration during time e.g. classification, centrifugal and gravity collection or separation, where it is often necessary to determine the trajectories of particle accelerating in a fluid for proposes of design or improved operation $[15,16]$. Unfortunately, there are few studies in the literature in the filled of rolling particles and the major part of the available investigations are related to the use of a rolling ball viscometer to measure viscosity of liquids [5,6]. Hasan [7] studied the role of wall effect on the rolling velocity of spherical particles in Newtonian media. He found a very limited correlation for $(\mathrm{d} / \mathrm{D})>0.707$ as follows:

$$
C_{D}=\frac{15.717}{\operatorname{Re}}\left(1-\left(\frac{d}{D}\right)\right)^{-2.5}
$$

Where, Reynolds number is defined as follow:

$$
\operatorname{Re}=\frac{\rho . u . D}{\mu}
$$

Where Re, d, and D, are the particle Reynolds number, particle diameter and tube inner diameter respectively. Chhabra et al. [12] presented a valuable experimental work for drag on spheres in rolling motion in inclined smooth tubes. They used an enough number of sphere made of glass and steel with four smooth walled glass tubes of different diameter. They used numerous aqueous solutions of glycerol and glucose syrup to cover a wide range of Reynolds number. The angles of inclination and sphere-to-tube diameter ratios were varied from 3 to 30 and 0.114 to 0.58 , respectively. Therefore, the Reynolds number range was $10^{-6}<\operatorname{Re}<3000$. They had 900 data points to define their empirical correlations. It was concluded that the sphere-to-wall diameter ratio (d/D), is not a significant parameter at the $95 \%$ confidence level. Consequently, the authors presented a three-part equation as follows:

$$
\begin{aligned}
& C_{D}=\frac{225}{\operatorname{Re}}, \operatorname{Re}<1 \\
& C_{D}=1+\frac{235}{\operatorname{Re}}, 1 \leq \operatorname{Re} \leq 250 \\
& C_{D}=1.35+\frac{177.5}{\operatorname{Re}}, 250 \leq \operatorname{Re}
\end{aligned}
$$


Eqs. (3-5) predict the transition points within about 5\%, and correlates the experimental set of data within an average error of $8 \%$. To describe a general correlation covering the experimental data we describe a new correlation using Chhabra et al. experimental points [12] as:

$$
C_{D}=1.2+\frac{190}{\operatorname{Re}}+\frac{1.003 \times 10^{-7}}{\operatorname{Re}^{2}}, 10^{-6} \leq \operatorname{Re} \leq 3000
$$

The third term in the right hand side of the Eq. (6) is important in low Reynolds number and its effect vanish by increasing of the Reynolds number and reduction of the drag coefficient. Eq. (6) is in very good agreement with results of the reference [12] and correlates the data with average error of $8.4883 \%$. The maximum difference between values of Eqs. (5) and (6), and experimental data, is related to the transient region where the Reynolds number is in the range of $5.8 \times 10^{-2} \leq \operatorname{Re} \leq 4.5$. Aside from mentioned work of Chhabra et al. [12], all other surveys of the rolling motion of the particles are related to open channels [12-15]. In reality, when a sphere is rolling in a tube, the wall exerts an extra retardation effect on it due to upward motion of the fluid through the eccentric annular gap between the particle and the wall this issue distinguishes the mechanism of rolling in tubes from open channels. It is clean that a few studies are performed on rolling motion of particles, especially in tubes while it is an important practical issue both in nature and industry. Moreover, most of the previous studies in particles motion and sedimentations are experimental or numerical. However, an exact analytical expression is more opportune for engineering calculations, and is also the evident starting point for a better understanding of the relationship between the physical properties of the sphere-fluid combination and the accelerated motion of the sphere. In addition, In contrast to steady-state motion of particles much less has been reported about the acceleration motion of spherical particles in incompressible Newtonian fluids. The accelerated motion is relevant to many processes such as particle classification, centrifugal and gravity particle collection and/or separation, where it is often necessary to determine the trajectories of particles accelerating in a fluid [14]. Furthermore, for other particular situations, like viscosity measurement using the falling-ball method or raindrop terminal velocity measurement it is necessary to know the time and distance required for particles to reach their terminal velocities. In this case study, similarity transformation has been used to reduce the governing differential equations into an ordinary non-linear differential equation. In most cases, these problems do not admit analytical solution, so these equations should be solved using special techniques. The differential transform method is based on Taylor expansion. It constructs an analytical solution in the form of a polynomial. It is different from the traditional high order Taylor series method, which requires symbolic computation of the necessary derivatives of the data functions. The Taylor series method is computationally taken long time for large orders. The differential transform is an iterative procedure for obtaining analytic Taylor series solutions of differential equations. Differential transform has the inherent ability to deal with nonlinear problems, and consequently Chiou [17] applied the Taylor transform to solve non-linear vibration problems. Furthermore, the method may be employed for the solution of both ordinary and partial differential equations. Jang et al. [18] applied the twodimensional differential transform method to the solution of partial differential equations. Finally, Hassan [19] adopted the Differential Transformation Method to solve some problems. The method was successfully applied to various practical problems [20-21].

The aim of current study is the analytically investigation of acceleration motion of a spherical particle rolling down an inclined boundary with drag coefficient in form of Eq. (3), using the Differential Transformation Method (DTM). Investigation and solution of falling objects' equation is a new application for DTM which was used for some other engineering problems. 


\section{PROBLEM DEFINITION}

Consider a spherical particle of diameter $\mathrm{d}$ and density of $\rho_{\mathrm{s}}$ rolling down a smooth tube having angle of inclination a with the horizontal, and filled with an incompressible Newtonian fluid of density $\rho$ Let $u$ represent the velocity of the sphere at any instant $t$ and $g$ the acceleration due to gravity. Figure. 1 illustrates a schematic view of the present problem.

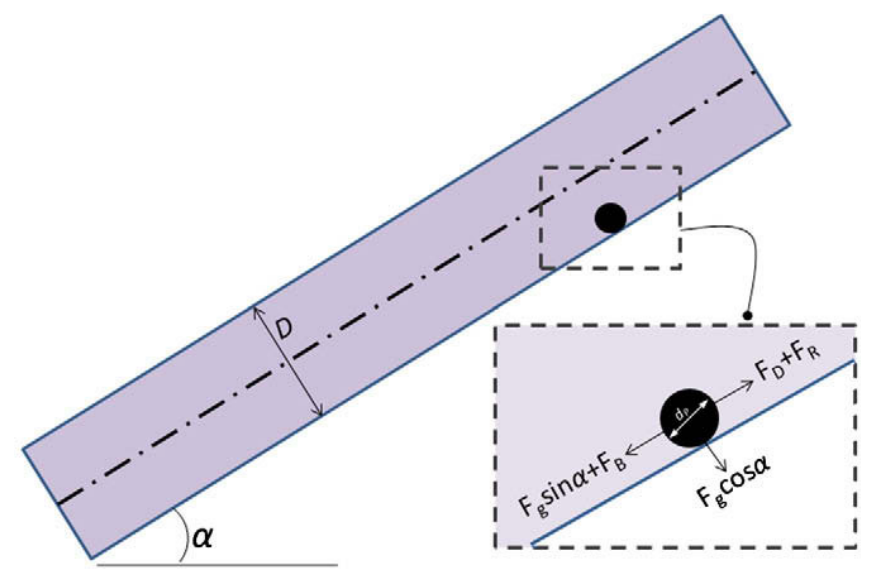

Figure.1 a schematic figure of current problem [15]

The forces acting on the spheres are the fluid-drag, $F_{D}$, fluid lift force, $F_{L}$, buoyancy force, $F_{B}$, gravitational force, $F_{g}$, solid-solid resistance force due to rolling, $F_{R}$, virtual mass force, $F_{V M}$ due to relative acceleration of the fluid around the particle. It is reasonable to postulate that since smooth walled tubes have been studied, $F_{R}$ is expected to be negligible (or alternately $F_{D}+F_{R}$ can be regarded as the total resistance to sphere motion, which is included in the drag coefficient). The all of detail of problem was explained at [15].The equation of motion is gained as follow from [15]:

$\frac{\pi d^{3}}{6} \rho_{s}\left(1.4+20 \frac{\rho}{\rho_{s}}\right) \frac{d u}{d t}=\frac{\pi d^{3}}{6} \rho_{s} g\left(1 \frac{\rho}{\rho_{s}}\right) \sin \alpha \frac{15 \pi d_{p}^{3} \rho}{100} u^{2}-2375 \pi d_{p} \mu u \frac{1.00 ख 10^{7} \pi \mu^{2}}{8 \rho_{c}}$

To simplify the post processes of the problem we had generated four coefficients in the equation above. Therefore, Eq. (7) is reduced to:

$$
a \frac{d^{2} w(t)}{d t^{2}}+b \frac{d w(t)}{d t}+c\left(\frac{d w(t)}{d t}\right)^{2}-d=0, w(0)=0, \quad \frac{d w(0)}{d t}=0
$$

Where:

$$
\begin{aligned}
& a=\frac{\pi \cdot d^{3}}{6} \rho_{s}\left(1.4+2.0 \frac{\rho}{\rho_{s}}\right) \\
& b=23.75 \pi \cdot d_{p} \cdot \mu \cdot \rho
\end{aligned}
$$


$c=\frac{15 \pi \cdot d_{p}^{3} \cdot \rho}{100}$

$d=\frac{\pi \cdot d^{3}}{6} \rho_{s} g\left(1-\frac{\rho}{\rho_{s}}\right) \sin \alpha-\frac{1.003 \times 10^{-7} \pi \mu^{2}}{8 \rho_{c}}$

With change of variation as bellow we obtain velocity,

$u(t)=\frac{d w(t)}{d t}$

By substituting Eq. (11) into Eq. (6) we will have:

$a \frac{d u(t)}{d t}+b u(t)+c(u(t))^{2}-d=0, \quad u(0)=0$

Eqs.(8) and (14) are non-linear ordinary differential equations which could be solved by numerical techniques such Runge-Kutta method. We employed DTM and compared our results with numerical solution of 4th order Runge-Kutta method using the Maple package.

\section{DIFFERENTIAL TRANSFORMATION METHOD}

We suppose $x(\tau)$ to be analytic function in a domain $D$ and $\tau=\tau_{i}$ represent any point in $D$. The function $x(\tau)$ is then represented by one power series whose center is located at $\tau_{i}$. The Taylor series expansion function of $x(\tau)$ is in the form of [23]:

$$
x(\tau)=\sum_{k=0}^{\infty} \frac{\left(\tau-\tau_{i}\right)^{k}}{k !}\left[\frac{d^{k} x(\tau)}{d t^{k}}\right]_{\tau=\tau_{i}}, \quad \forall \tau \in D
$$

The particular case of Eq. (13) when $\tau_{i}=0$ is referred to as the Maclaurin series of $x(\tau)$ and is expressed as:

$$
x(\tau)=\sum_{k=0}^{\infty} \frac{\tau^{k}}{k !}\left[\frac{d^{k} x(\tau)}{d \tau^{k}}\right]_{\tau=0}, \quad \forall \tau \in D
$$

As explained in [25-31] the differential transformation of the function $x(\tau)$ is defined as follows:

$X(k)=\sum_{k=0}^{\infty} \frac{H^{k}}{k !}\left[\frac{d^{k} x(\tau)}{d \tau^{k}}\right]_{\tau=0}$

Where, $x(\tau)$ is the original function and $X(k)$ is the transformed function. The differential spectrum of $X(k)$ is confined within the interval $\tau \in[0, H]$, where $H$ is a constant. The differential inverse transform of $X(k)$ is defined as follows: 
$x(\tau)=\sum_{k=0}^{\infty}\left(\frac{\tau}{H}\right)^{k} X(k)$

It is clear that the concept of differential transformation is based upon the Taylor series expansion. The values of function $X(k)$ at values of argument $k$ are referred to as discrete, i.e. $X(0)$ is known as the zero discrete, $X(1)$ as the first discrete, etc. The more discrete available, the more precise it is possible to restore the unknown function. The function $x(\tau)$ consists of the T-function $X(k)$, and its value is given by the sum of the T-function with $(\tau / H)^{k}$ as its coefficient. In real applications, at the right choice of constant $H$, the larger values of argument $k$ the discrete of spectrum reduce rapidly. The function $x(\tau)$ is expressed by a finite series and Eq. (16) can be written as:

$x(\tau)=\sum_{k=0}^{n}\left(\frac{\tau}{H}\right)^{k} X(k)$

Eq. (19) implies that the value $k=n+1 \rightarrow \infty$ is negligible.

If $u(t)$ and $v(t)$ are two uncorrelated functions with time $t$ where $U(k)$ and $V(k)$ are the transformed functions corresponding to $u(t)$ and $v(t)$ then we can easily proof the fundamental mathematics operations executed by differential transformation. The fundamental mathematical operations performed by differential transformation method are listed in Table 1 [25-30].

\begin{tabular}{|c|c|}
\hline Original function & Transformed function \\
\hline$x(t)=\alpha f(t) \pm \beta g(t)$ & $X(k)=\alpha F(k) \pm \beta G(k)$ \\
\hline$x(t)=\frac{d f(t)}{d t}$ & $X(k)=(k+1) F(k+1)$ \\
\hline$x(t)=\frac{d^{2} f(t)}{d t^{2}}$ & $X(k)=(k+1)(k+2) F(k+2)$ \\
\hline$x(t)=t^{m}$ & $X(k)=\delta(k-m)= \begin{cases}1 & k=m \\
0 & k \neq m\end{cases}$ \\
\hline$x(t)=f(t) g(t)$ & $X(k)=\sum_{l=0}^{k} F(l) G(k-l)$ \\
\hline
\end{tabular}

\section{APPLICATION OF DIFFERENTIAL TRANSFORMATION METHOD}

Now we apply Differential Transformation Method into Eq. (8) for find $w(t)$ as displacement. Taking the differential transform of Eq. (16) with respect to $t$ according table 1 gives: 


$$
\begin{aligned}
& a\left((k+2)(k+1) \mathrm{W}_{\mathrm{k}+2}\right)+b\left((k+1) \mathrm{W}_{\mathrm{k}+1}\right)+c\left(\sum_{j=0}^{k}(k-j+1) \mathrm{W}_{\mathrm{k}-\mathrm{j}+1}(j+1) \mathrm{W}_{\mathrm{j}+1}\right) \\
& -d\left(\left\{\begin{array}{ll}
1 & \mathrm{k}=0 \\
0 & \text { otherwise }
\end{array}\right)=0\right.
\end{aligned}
$$

By suppose $W_{0}$ and $W_{1}$ are apparent from boundary conditions by solving Eq. (20) respect $W_{k+2}$, we will have:

$$
\begin{aligned}
& W_{2}=-\frac{1}{2} \frac{\left(b W_{1}+c W_{1}^{2}-d\right)}{a} \\
& W_{3}=-\frac{1}{3} \frac{\left(W_{2}\left(b+2 c W_{1}\right)\right)}{a} \\
& W_{4}=\frac{-1}{12} \frac{\left(3 b W_{3}+6 c W_{3} W_{1}+4 c W_{2}^{2}\right)}{a} \\
& W_{5}=-\frac{1}{5} \frac{\left(b W_{4}+2 c W_{4} W_{1}+3 c W_{3} W_{2}\right)}{a} \\
& \text {. }
\end{aligned}
$$

The above process is continuous. Substituting Eq. (20-24) into the main equation based on DTM, Eq. (19), it can be obtained the closed form of the solutions,

$$
\begin{aligned}
& w(t)=W_{0}+t W_{1}-\frac{t^{2}}{2} \frac{\left(b W_{1}+c W_{1}^{2}-d\right)}{a}-\frac{t^{3}}{3} \frac{\left(W_{2}\left(b+2 c W_{1}\right)\right)}{a}-\frac{t^{4}}{12} \frac{\left(3 b W_{3}+6 c W_{3} W_{1}+4 c W_{2}^{2}\right)}{a} \\
& -\frac{\mathrm{t}^{5}}{5} \frac{\left(b W_{4}+2 c W_{4} W_{1}+3 c W_{3} W_{2}\right)}{a}+\Lambda
\end{aligned}
$$

Substituting Eq. (21-24) into the main equation based on DTM, it can be obtained the closed form of the solutions. In this stage for achieve higher accuracy we use sub-domain technique, i.e. the domain of $t$ should be divided into some adequate intervals and the values at the end of each interval will be the initial values of next one. For example for first sub-domain assume that distance of each interval is 0.005 . For first interval, $0 \rightarrow 0.005$ boundary conditions are From boundary conditions in Eq. (8) at point $t=0$. By exerting transformation, we will have:

$$
W_{0}=0
$$

The other boundary conditions are considered as follow:

$$
W_{1}=0
$$

As mentioned above for next interval, $0.005 \rightarrow 0.01$, new boundary conditions are:

$$
W_{0}=w(0.005)
$$

The next boundary condition is considered as follow: 


$$
W_{1}=\frac{d w}{d t}(0.2)
$$

For this interval function $w(t)$ is represented by power series whose center is located at 0.005 , by means that in this power series $t$ convert to $(t-0.005)$.

As we can see bellow in similar case for achieves the solution for $u(t)$ as velocity we should apply DTM on Eq. (14) to find transformed function.

$$
a\left((k+1) \mathrm{U}_{\mathrm{k}+1}\right)+b \mathrm{U}_{\mathrm{k}}+c\left(\sum_{j=0}^{k} \mathrm{U}_{k-j} \mathrm{U}_{\mathrm{j}}\right)-d\left(\left\{\begin{array}{ll}
1 & \mathrm{k}=0 \\
0 & \text { otherwise }
\end{array}\right)=0\right.
$$

By assuming that $U_{0}$ is apparent from boundary condition by solving Eq. (30) respect $U_{k+1}$, we will have:

$$
\begin{aligned}
& U_{1}=-\frac{\left(b U_{0}+c U_{0}{ }^{2}-d\right)}{a} \\
& U_{2}=-\frac{1}{2} \frac{\left(U_{1}\left(b+2 c U_{0}\right)\right)}{a} \\
& U_{3}=-\frac{1}{3} \frac{\left(b U_{2}+2 c U_{2} U_{0}+c U_{1}^{2}\right)}{a} \\
& U_{4}=-\frac{1}{4} \frac{\left(b U_{3}+2 c U_{3} U_{0}+2 c U_{2} U_{1}\right)}{a} \\
& U_{5}=-\frac{1}{5} \frac{\left(b U_{4}+2 c U_{4} U_{0}+2 c U_{3} U_{1}+c U_{2}^{2}\right)}{a}
\end{aligned}
$$

As mentioned above this process is continuous. By substituting Eq. (31-35) into Eq. (19), closed form of the solutions is,

$$
\begin{aligned}
& u(t)=U_{0}-t \frac{\left(b U_{0}+c U_{0}^{2}-d\right)}{a}-\frac{t^{2}}{2} \frac{\left(U_{1}\left(b+2 c U_{0}\right)\right)}{a}-\frac{t^{3}}{3} \frac{\left(b U_{2}+2 c U_{2} U_{0}+c U_{1}^{2}\right)}{a} \\
& -\frac{t^{4}}{12} \frac{\left(b U_{3}+2 c U_{3} U_{0}+2 c U_{2} U_{1}\right)}{a}-\frac{t^{5}}{5} \frac{\left(b U_{4}+2 c U_{4} U_{0}+2 c U_{3} U_{1}+c U_{2}^{2}\right)}{a}+\Lambda
\end{aligned}
$$

And for achieve higher accuracy we use sub-domain technique as described above. By substituting Eqs. (9-12) into Eq. (25) and Eq. (36), an exact solution for $w(t)$ and $u(t)$ can be obtained which is only related to the particle and the fluid properties. 


\section{RESULTS AND DISCUSSION}

The mentioned method was applied for real combination of solid-fluid. A single Aluminum spherical particle with versus diameter was assumed to roll down a smooth inclined plane in an infinity medium of Ethylene-glycol, glycerin solution and water. Required physical properties of selected materials are given in Table 2 .

\begin{tabular}{|c|c|c|}
\hline Table 2 Physical properties of materials & & \\
Material & Density & Viscosity \\
\hline Water & 996.51 & 0.001 \\
\hline Ethylene-glycol & 1111.40 & 0.0157 \\
\hline Glycerin & 1259.90 & 0.779 \\
\hline Aluminum & 2702.0 & - \\
\hline
\end{tabular}

In the modeling, Aluminum with density of $\rho_{s}=2702.00 \mathrm{~kg} / \mathrm{m}^{3}$ is used for dispersed phase (particle).Inserting above properties into Eqs.(9) to (12), different combinations are gained which are classified in Table 3.

\begin{tabular}{|c|c|c|c|c|c|}
\hline \multicolumn{6}{|c|}{ Table 3 Coefficients for different liquid combinations } \\
\hline Fluid & $\mathrm{d}(\mathrm{mm})$ & $c^{a}$ & B & $\mathrm{C}$ & $\mathrm{D}$ \\
\hline Water & 1 & 50.000003024123088 & 0.000074610625 & 0.0004695804248 & $7.638667428 \mathrm{e}-7$ \\
\hline & & 1: 0.000003024123088 & 0.000074610625 & 0.0004695804248 & 0.000002268242935 \\
\hline & & 2. 0.000003024123088 & 0.000074610625 & 0.0004695804248 & 0.000003283167712 \\
\hline & & 310.000003024123088 & 0.000074610625 & 0.0004695804248 & 0.000004382231328 \\
\hline & 2 & 1: 0.00002419298470 & 0.000149221250 & 0.001878321699 & 0.00001814594348 \\
\hline & 3 & 1: 0.00008165132337 & 0.000223831875 & 0.004226223822 & 0.00006124255925 \\
\hline Ethylene & 1 & 2. 0.000003144432067 & 0.001171386812 & 0.0005237194650 & 0.000003061997753 \\
\hline Glycerin & 1 & 2. 0.000003299936317 & 0.05961388938 & 0.0005936963775 & 0.000002776106643 \\
\hline
\end{tabular}

By substituting above coefficients in Eq. (8), and for four different inclination angles, twelve different nonlinear equations are achieved. Inclination angles were selected to be $5^{\circ}, 15^{\circ}, 22^{\circ}$ and $30^{\circ}$. Differential Transformation Method was applied to gained equations and results were compared with numerical method. The influence of particle size is studied where the diameter of the particles is varied in the range of $1 \mathrm{~mm}<d_{p}<3 \mathrm{~mm}$. Figs. ( $\left.2-4\right)$ shown the variations of the displacement and velocity and acceleration for three different particles rolling in a tube inclined with the angle of $15^{\circ}$ and filled with the water.

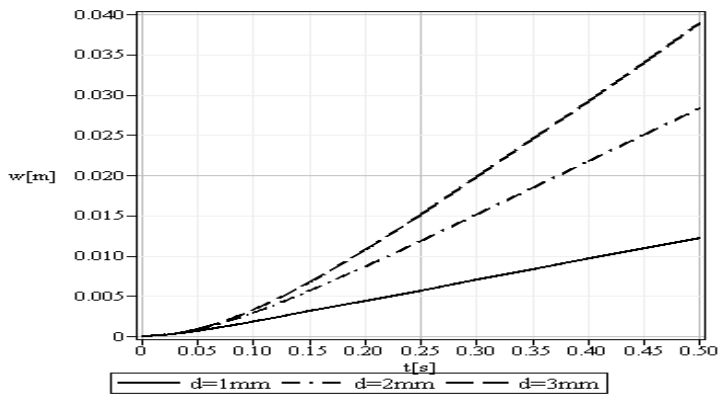

Figure.2 displacement variation for three spherical particles rolling in a tube filled with the water 


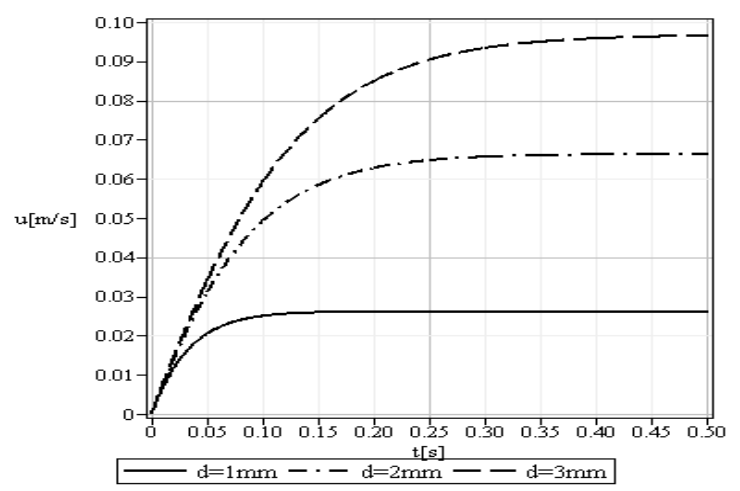

Figure. 3 velocity variation for three spherical particles rolling in a tube filled with the water

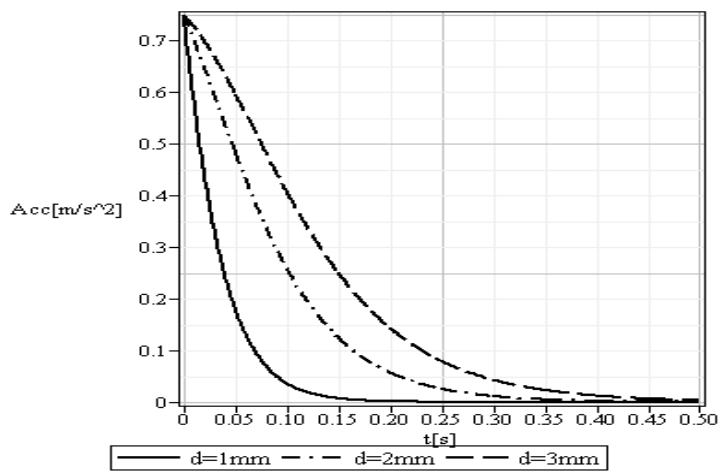

Figure.4 Acceleration variation for three spherical particles rolling in a tube filled with the water

These figures clearly illustrate that how different diameters affect the displacement and velocity and acceleration of particles while other conditions are equivalent. Observably, it is shown that the value of the displacement and velocity and acceleration in a rolling procedure is significantly increased with adding to the particle size. The variation of displacement and velocity and acceleration of the particle versus time for the different inclination angles are shown in Figs. (57).

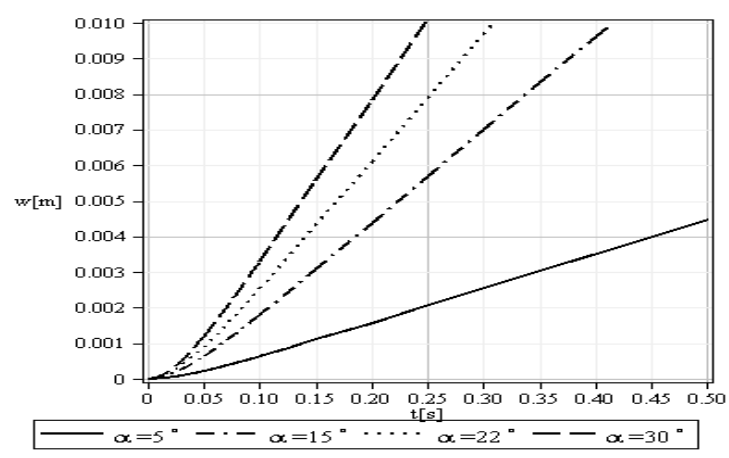

Figure. 5 displacement variation of a spherical particle rolling in a tube for different angles 


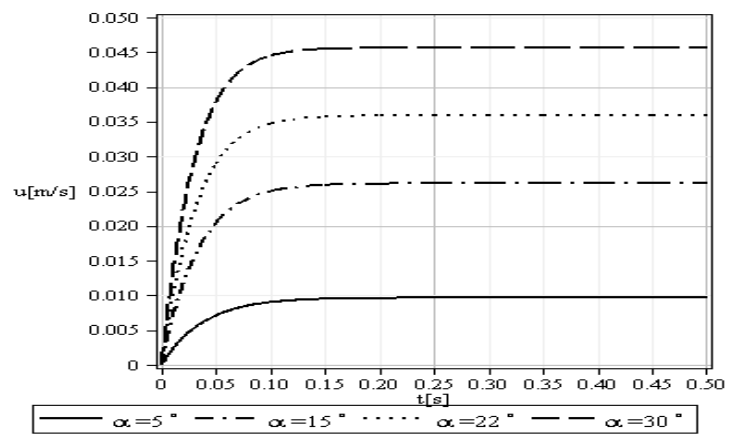

Figure.6 Velocity variation of a spherical particle rolling in a tube for different angles

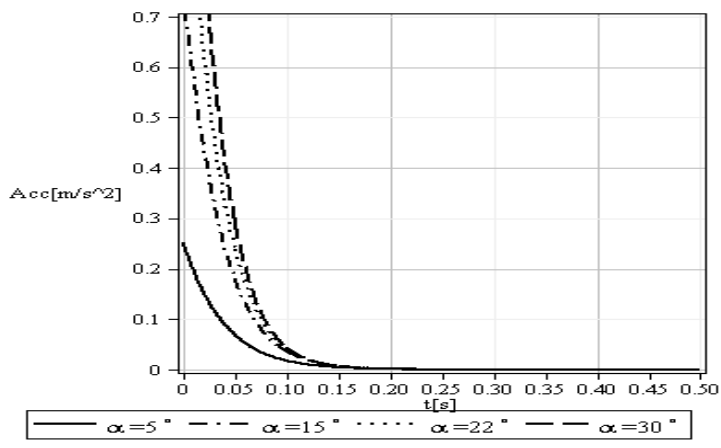

Figure.7 Acceleration variation of a spherical particle rolling in a tube for different angles

For a given the fluid viscosity, by increasing inclination angle, displacement and acceleration duration are increasing. Results show that increasing of inclination angle increases the terminal velocity as well as acceleration and displacement. Outcomes illustrated that higher acceleration is obtained for larger inclination angle. Variable displacement and velocity for sphere which its fluid is water, results of the present analysis are tabulated and comprised with the numerical solution obtained by fourth-order Runge-Kutta method in Table 4 and 5 .

\begin{tabular}{|c|c|c|c|}
\hline \multicolumn{4}{|c|}{ Table 4 the $u(t)$ obtained from DTM and NS for water, $\alpha=15^{\circ}, \mathrm{d}=1 \mathrm{~mm}$} \\
\hline t & $\mathbf{U}_{\text {DTM }}$ & $\mathbf{U}_{\mathrm{NS}}$ & $\begin{array}{l}\text { Absolute } \\
\text { Error }\left(\mathrm{U}_{\mathrm{DTM}^{-}} \mathrm{U}_{\mathrm{NS}}\right)\end{array}$ \\
\hline $\mathbf{0}$ & 0 & 0 & 0 \\
\hline 0.05 & 0.02047928110 & 0.02047930816 & 2.70611E-08 \\
\hline 0.1 & 0.02499292820 & 0.02499294991 & $2.17 \mathrm{E}-08$ \\
\hline 0.15 & 0.02589242595 & 0.02589245023 & 2.42859E-08 \\
\hline 0.2 & 0.02606797450 & 0.02606799339 & 1.88962E-08 \\
\hline 0.25 & 0.02610209443 & 0.02610211188 & $1.74534 \mathrm{E}-08$ \\
\hline 0.3 & 0.02610872071 & 0.02610873515 & $1.44461 \mathrm{E}-08$ \\
\hline 0.35 & 0.02611000738 & 0.02611002114 & $1.38 \mathrm{E}-08$ \\
\hline 0.4 & 0.02611025723 & 0.02611026728 & $1.01 \mathrm{E}-08$ \\
\hline 0.45 & 0.02611030573 & 0.02611031057 & 4.84E-09 \\
\hline 0.5 & 0.02611031515 & 0.02611032008 & 4.93E-09 \\
\hline
\end{tabular}




\begin{tabular}{|llll|}
\hline \multicolumn{5}{|c|}{ Table $\mathbf{5}$ the $w(t)$} & obtained from DTM and NS for $\begin{array}{l}\text { water, } \alpha=15^{\circ}, \mathrm{d}=1 \mathrm{~mm} \\
\mathbf{T}\end{array}$ & $\mathbf{W}_{\text {DTM }}$ & $\mathbf{W}_{\text {NS }}$ & $\begin{array}{l}\text { Absolute } \\
\text { Error }\left(\mathbf{W}_{\text {DTM}^{-}} \mathbf{W}_{\text {NS }}\right)\end{array}$ \\
\hline $\mathbf{0}$ & 0 & 0 & 0 \\
\hline $\mathbf{0 . 0 5}$ & 0.000629372276 & 0.000629371444 & $8.32 \mathrm{E}-10$ \\
\hline $\mathbf{0 . 1}$ & 0.001794953256 & 0.001794952721 & $5.35 \mathrm{E}-10$ \\
\hline $\mathbf{0 . 1 5}$ & 0.003072942044 & 0.003072941443 & $6.01 \mathrm{E}-10$ \\
\hline $\mathbf{0 . 2}$ & 0.004373099347 & 0.004373098915 & $4.32 \mathrm{E}-10$ \\
\hline $\mathbf{0 . 2 5}$ & 0.005677574236 & 0.005677573848 & $3.88 \mathrm{E}-10$ \\
\hline $\mathbf{0 . 3}$ & 0.006982887961 & 0.006982887666 & $2.95 \mathrm{E}-10$ \\
\hline $\mathbf{0 . 3 5}$ & 0.008288364581 & 0.008288364307 & $2.74 \mathrm{E}-10$ \\
\hline $\mathbf{0 . 4}$ & 0.009593872830 & 0.009593872669 & $1.61 \mathrm{E}-10$ \\
\hline $\mathbf{0 . 4 5}$ & 0.01089938727 & 0.010989387220 & $5 \mathrm{E}-11$ \\
\hline 0.5 & 0.01220490284 & 0.012204902801 & $3.9 \mathrm{E}-11$ \\
\hline
\end{tabular}

Presented results demonstrate an excellent agreement between DTM and numerical solution. In Figs. $(8,9)$ the agreement between DTM and numerical solution for displacement and velocity of Eq.(8) when the fluid is water, $\alpha=15^{\circ}, d_{p}=1 \mathrm{~mm}$ is shown.

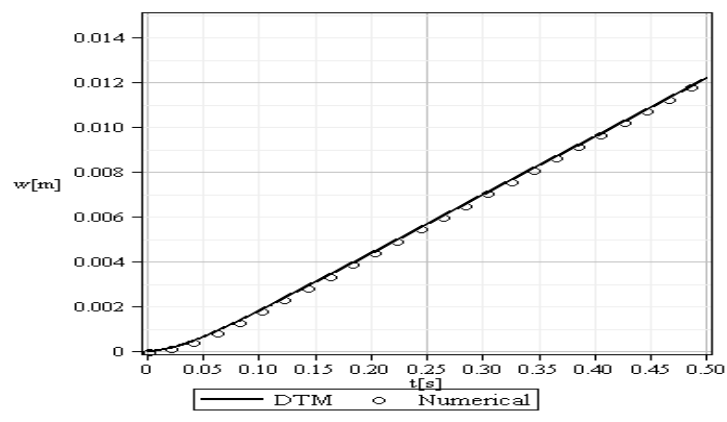

Figure.8 DTM and numerical solutions of Eq. (8) when the fluid is water, $\alpha=15^{\circ}, \mathrm{d}=1 \mathrm{~mm}$

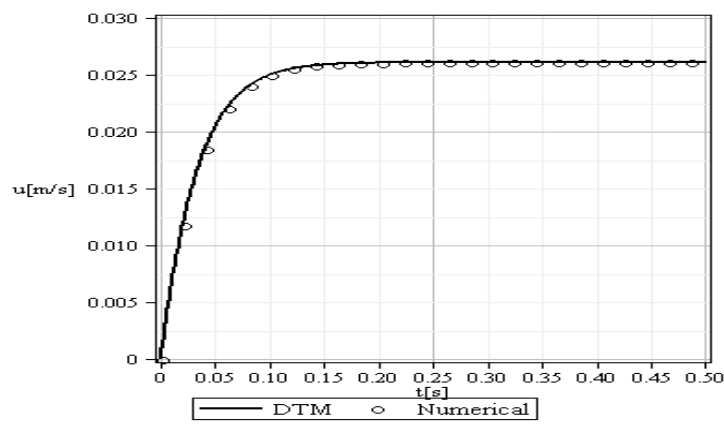

Figure.9 DTM and numerical solutions of Eq. (8) when the fluid is water, $\alpha=15^{\circ}, \mathrm{d}=1 \mathrm{~mm}$

In this case, a very interesting agreement between the results of two methods is observed which confirms the excellent validity of the DTM. 


\section{CONCLUSIONS}

In this paper, Differential Transformation Method (DTM) is applied to obtain the solution of the unsteady motion of a spherical particle rolling down an inclined tube in a Newtonian fluid. Equation was solved generally and for some real combinations of solid-liquid. Instantaneous velocity, acceleration and position were obtained as results and outcomes were compared with Runge-Kutta method solution. Very good agreement has been seen between numerical and current analytical method. Results show that for a given condition of particle and fluid, an increase in inclination angle, $\alpha$, results in an increase in terminal displacement and velocity and acceleration. Current work approved the simplicity and capability of Differential Transformation Method. Solution of equation of motion for an object rolling down an inclined boundary is a new application of DTM and could be used in wide area of scientific problems, especially hydraulic and sedimentation engineering.

\section{REFERENCES}

[1] J.W. Delleur, New results and research needs on sediment movement in urban drainage, J. Water Resour. Plan. Manage, ASCE; 186-19( 2001).

[2] T. Hvitved-Jacobsen, J. Vollertsen, N. Tanaka, Wastewater quality changes during transport in sewers: An integrated aerobic and anaerobic model concept for carbon and sulfur microbial transformations, Water Sci. Technol. 257-264(1998).

[3] Z.X. Cao, Equilibrium near-bed concentration of suspended sediment, J. Hydraul. Eng., ASCE; 12701278(1999).

[4] J.S. Bridge, S.J. Bennett, A model for the entrainment and transport of sediment grains of mixed sizes, shapes, and densities, Water Resour. Res., 337-363(1992).

[5] J. Duran, The physics of fine powders: Plugging and surface instabilities, C.R. Phys.,17-227(2002).

[6] J.G. Yates, Fundamentals of fluidized-bed processes, Butterworths, London, (1983).

[7] M.A. Hasan, The role of wall effects on the rolling velocity of spheres in Newtonian fluids, Chemical Engineering Journal 33 -97(1986).

[8] J. L. Boillat, N.H. Graf, Vitesse de sedimentation de particules spheriques en milieu turbulent, J. Hydraul. Res. 395-413(1982).

[9] D.D. Joseph, Y.L. Liu, M. Poletto, J. Feng, Aggregation and dispersion of spheres falling in viscoelastic liquids, J. Non-Newtonian Fluid mech, 54: 45-86 (1994).

[10] R. Clift, J.R. Grace, M.E. Weber, Bubbles, Drops and Particles, Academic Press, New York, (1978).

[11] A.R. Khan, J.F. Richardson, The resistance to motion of a solid sphere in a fluid, Chem. Eng. Commun.; 135- 150(1987).

[12] R.P. Chhabra, J.M. Ferreira, An analytical study of the motion of a sphere rolling down a smooth inclined plane in an incompressible Newtonian fluid, Powder Technology ,130-138(1999).

[13] M. Hartman, J.G. Yates, Free-fall of solid particles through fluids, Collect. Czechoslov. Chem. Commun. 961-982(1993).

[14] J.M. Ferreira, R.P. Chhabra, Accelerating motion of a vertically falling sphere in incompressible Newtonian media: an analytical solution, J. Powder Technology, 97: 6-15(1998).

[15] C.D. Jan, J.C. Chen, Movements of a sphere rolling down an inclined plane. J. Hydraulic Res., 689706(1997).

[16] J.S. Chiou, J.R. Tzeng, Application of the Taylor transform to nonlinear vibration problems, Transaction of the American Society of Mechanical Engineers, J. Vib.Acoust. (83-87)( 1996).

[17] C.K. Chen, S.P. Ju, Application of differential transformation to transient advective-dispersive transport equation, Appl. Math. Comput. 25-38(2004).

[18] Y.L. Yeh, C.C. Wang, M.J. Jang, Using finite difference and y to analyze of large deflections of orthotropic rectangular plate problem, Appl. Math. Comput. 1146-1156(2007).

[19] I.H. Abdel-Halim Hassan.: Differential transformation technique for solving higher-order initial value problems. Appl. Math. Comput. 299-311(2004).

[20] M. Jalaal, D.D. Ganji, An analytical study on motion of a sphere rolling down an inclined plane submerged in a Newtonian fluid, Powder Technology, 82-92(2010). 
[21] M. Jalaal, D.D. Ganji, G. Ahmadi, Analytical investigation on acceleration motion of a vertically falling spherical particle in incompressible Newtonian media, Advanced Powder Technology (2009).

[22] M. Jalaal, D.D. Ganji, On unsteady rolling motion of spheres in inclined tubes filled with incompressible Newtonian fluids, Advanced Powder Technology (2010). 